Discrete Comput Geom 31:327-335 (2004)

DOI: $10.1007 / \mathrm{s} 00454-003-2868-\mathrm{y}$

\title{
The Extreme Points of Subsets of $s$-Concave Probabilities and a Geometric Localization Theorem
}

\author{
Matthieu Fradelizi ${ }^{1}$ and Olivier Guédon ${ }^{2}$ \\ ${ }^{1}$ Laboratoire d'Analyse et de Mathématiques Appliquées, Université de Marne-la-Vallée, \\ 5 Bd Descartes, Champs-sur-Marne, 77454 Marne-la-Vallée Cedex 2, France \\ fradeliz@math.univ-mlv.fr \\ ${ }^{2}$ Institut de Mathématiques, Université Pierre et Marie Curie, \\ boîte 186, 4 Place Jussieu, 75005 Paris, France \\ guedon@ccr.jussieu.fr
}

\begin{abstract}
We prove that the extreme points of the set of $s$-concave probability measures satisfying a linear constraint are some Dirac measures and some $s$-affine probabilities supported by a segment. From this we deduce that the constrained maximization of a convex functional on the $s$-concave probability measures is reduced to this small set of extreme points. This gives a new approach to a localization theorem due to Kannan, Lovász and Simonovits which happens to be very useful in geometry to obtain inequalities for integrals like concentration and isoperimetric inequalities. Roughly speaking, the study of such inequalities is reduced to these extreme points.
\end{abstract}

\section{Introduction}

The localization lemma of Lovász and Simonovits [7] indicates that if one wants to prove an inequality for all $1 / n$-concave measures on $\mathbb{R}^{n}$, it is enough to test this inequality over all $1 / n$-affine measures supported by a segment. This may reduce the problem considerably. The precise statement is as follows:

Theorem [7]. Let $f$ and $g$ be two lower semi-continuous Lebesgue integrable functions on $\mathbb{R}^{n}$ such that

$$
\int_{\mathbb{R}^{n}} f(x) d x>0 \text { and } \int_{\mathbb{R}^{n}} g(x) d x>0 .
$$

Then there exists $a, b \in \mathbb{R}^{n}$ and an affine function $\ell:[0,1] \rightarrow \mathbb{R}^{+}$such that

$$
\int_{0}^{1} f((1-t) a+t b) \ell(t)^{n-1} d t>0 \quad \text { and } \quad \int_{0}^{1} g((1-t) a+t b) \ell(t)^{n-1} d t>0 .
$$


The proof given in [7] consists in using infinitely many times the "bisection method" in an algorithmic procedure to perform the construction of a sequence of measures which converges to a $1 / n$-affine measure supported by a segment satisfying the same inequalities. In [6] Kannan et al. deduce from this result a theorem in terms of products of integrals over convex sets (see Corollary 1 below) that they can extend to the case of log-concave measures.

In this paper we generalize the localization lemma of Lovász and Simonovits [7] to the $s$-concave measures on $\mathbb{R}^{n}$ for $-\infty \leq s \leq \frac{1}{2}$. The case $s=0$ corresponds to the log-concave measures. The definitions and well-known results about such measures are recalled in Section 2 of the paper. Our method consists in replacing the algorithmic procedure by the use of the Krein-Milman theorem. More precisely, in Theorem 1 we describe the extreme points of the convex hull of the set of all $s$-concave probabilities $\mu$ supported in a compact subset of $\mathbb{R}^{n}$ satisfying the constraint $\int f d \mu \geq 0$, where $f$ is a fixed upper semi-continuous function. Here again, the "bisection method" is at the heart of the proof to decide which probabilities are extreme in this set. Then a standard use of the Krein-Milman theorem shows that the supremum over this set of any convex functional is attained at these extreme points, i.e. either at Dirac measures or at some $s$-affine probabilities supported by a segment, this is our Theorem 2 . An easy example is to consider the functional $\mu \mapsto \int g d \mu$ for an upper semi-continuous function $g$ and we recover the conclusion of Lovász and Simonovits for two integrals.

The main results of this paper explained above are contained in Section 3, while the proof of the main theorem is postponed to Section 4. In Section 5 of the paper we prove a generalization to the case of several constraints. Such a generalization is announced to be true by Kannan et al. in [6]. In this part we use Borsuk's theorem to find an affine subspace which simultaneously bisects several integrals.

This localization principle is very powerful to obtain dimension-free inequalities. Under different constraints, it is the main tool to prove concentration inequalities [7], [5], general isoperimetric inequalities [6] and also to study distributional inequalities for polynomials over convex bodies in $\mathbb{R}^{n}[1]$, [4], [8].

\section{Preliminaries on $s$-Concave Measures on $\mathbb{R}^{n}$}

Given subsets $A$ and $B$ of the Euclidean $n$-space $\mathbb{R}^{n}$ and $\lambda>0$, we set $A+B=$ $\{x+y ; x \in A, y \in B\}$ and $\lambda A=\{\lambda x ; x \in A\}$. For a convex set $C$, we denote by $\mathcal{E} \operatorname{xt}(C)$ the set of extreme points of $C$. For all $s \in[-\infty,+\infty]$, we say in this paper that a measure $\mu$ in $\mathbb{R}^{n}$ is $s$-concave if the inequality

$$
\mu(\lambda A+(1-\lambda) B) \geq\left[\lambda \mu^{s}(A)+(1-\lambda) \mu^{s}(B)\right]^{1 / s}
$$

holds for all compact subsets $A, B \subset \mathbb{R}^{n}$ such that $\mu(A) \mu(B)>0$ and all $\lambda \in[0,1]$. The limit cases are interpreted by continuity. Thus the right-hand side of this inequality is equal to $\mu^{\lambda}(A) \mu^{1-\lambda}(B)$ for $s=0$, to $\min (\mu(A), \mu(B))$ for $s=-\infty$, and to $\max (\mu(A), \mu(B))$ for $s=+\infty$. Notice that an $s$-concave measure is $t$-concave for all $t \leq s$. We denote by $\mathcal{P}(K)$ the set of probabilities in $\mathbb{R}^{n}$ supported in a convex compact $K$. For a probability $\mu, \operatorname{supp}(\mu)$ denotes its support. 
For $\gamma \in[-\infty,+\infty]$, a function $f: \mathbb{R}^{n} \rightarrow \mathbb{R}^{+}$is $\gamma$-concave if the inequality

$$
f(\lambda x+(1-\lambda) y) \geq\left[\lambda f^{\gamma}(x)+(1-\lambda) f^{\gamma}(y)\right]^{1 / \gamma}
$$

holds for all $x$ and $y$ such that $f(x) f(y)>0$ and all $\lambda \in[0,1]$, where the limit cases are also interpreted by continuity. For example, the $-\infty$-concave functions are the quasiconcave ones (i.e. the functions $f$ such that $\{f \geq t\}$ is convex for all $t \in \mathbb{R}$ ). The link between the $s$-concave probabilities and the $\gamma$-concave functions is described in the work of Borell [3]. Notice that for $s>0$, his definition differs a little from ours because the inequality (*) was not restricted to the compact sets $A$ and $B$ such that $\mu(A) \mu(B)>0$. In particular, with our definition, for all $s \leq 1 / d$, there exists $s$-concave measures supported in an affine subspace of dimension $d$ and, for example, Dirac measures are $s$-concave for any $s \in[-\infty,+\infty]$.

Theorem [3]. Let $\mu$ be a measure in $\mathbb{R}^{n}$, let $G$ be the least affine subspace which contains the support of $\mu$, set $d=\operatorname{dim} G$ and let $m$ be the Lebesgue measure on $G$. Then for $-\infty \leq s \leq 1 / d$, $\mu$ is s-concave if and only if $d \mu=\psi d m$, where $0 \leq \psi \in L_{\mathrm{loc}}^{1}\left(\mathbb{R}^{n}, d m\right)$ and $\psi$ is $\gamma$-concave with $\gamma=s /(1-s d) \in[-1 / d,+\infty]$. Moreover, if $s>1 / d$, then $\mu$ is $s$-concave if and only if $\mu$ is a Dirac measure.

According to this theorem, we say that a measure $\mu$ is $s$-affine when its density $\psi$ satisfies that $\psi^{\gamma}$ (or $\log \psi$ if $s=\gamma=0$ ) is affine on its convex support with $\gamma=s /(1-s d)$. We will also need the following simple lemma.

Lemma 1. Let $K$ be a convex set in $\mathbb{R}^{n}, \gamma \in[-\infty, 1]$. If $C: K \rightarrow \mathbb{R}^{+}$is $\gamma$-concave and $V: K \rightarrow \mathbb{R}^{+}$is $\gamma$-affine, then $(C-V)_{+}: K \rightarrow \mathbb{R}^{+}$is $\gamma$-concave.

Proof. Let $\gamma \in(-\infty, 1] \backslash\{0\}$ and let $x$ and $y$ in $K$ be such that $C(x) \geq V(x)$ and $C(y) \geq V(y)$. By Minkowski's inequality for $\|\cdot\|_{\gamma}$ we know that for all $\lambda \in[0,1]$,

$$
\begin{gathered}
\left(\lambda C^{\gamma}(x)+(1-\lambda) C^{\gamma}(y)\right)^{1 / \gamma}-\left(\lambda V^{\gamma}(x)+(1-\lambda) V^{\gamma}(y)\right)^{1 / \gamma} \\
\geq\left(\lambda(C-V)^{\gamma}(x)+(1-\lambda)(C-V)^{\gamma}(y)\right)^{1 / \gamma}
\end{gathered}
$$

and as $C$ is $\gamma$-concave and $V$ is $\gamma$-affine, we get for all $x$ and $y$ in the support of $(C-V)_{+}$,

$$
(C-V)(\lambda x+(1-\lambda) y) \geq\left(\lambda(C-V)^{\gamma}(x)+(1-\lambda)(C-V)^{\gamma}(y)\right)^{1 / \gamma} .
$$

By sending $\gamma$ to 0 in Minkowski's inequality, we obtain the arithmetico-geometric inequality which gives the same conclusion for log-concave functions. The case $\gamma=-\infty$ is obvious.

\section{Results}

Our main result is the following theorem. 
Theorem 1. Let $n$ be a positive integer, let $K$ be a compact convex set in $\mathbb{R}^{n}$, let $s \in\left[-\infty, \frac{1}{2}\right]$ and let $f: K \rightarrow \mathbb{R}$ be an upper semi-continuous function. We denote by $P_{f}$ the set of s-concave probabilities supported in $K$ satisfying $\int f d \mu \geq 0$. The set of extreme points of $\operatorname{conv} P_{f}$ is exactly:

(1) the Dirac measures at points $x$ such that $f(x) \geq 0$,

(2) the probabilities $v$ which are s-affine, supported by a segment $[a, b] \subset K$, such that $\int f d v=0$ and $\int_{a}^{x} f d v>0$ on $(a, b)$ or $\int_{x}^{b} f d v>0$ on $(a, b)$.

Before proving Theorem 1, we show that it immediately extends the localization lemma of Lovász and Simonovits [7] and we prefer to see it as a constrained optimization problem on $s$-concave probabilities in $\mathbb{R}^{n}$.

Theorem 2. Under the same assumptions as in Theorem 1, if $\Phi: \mathcal{P}(K) \rightarrow \mathbb{R}$ is a convex upper semi-continuous function, then $\sup \left\{\Phi(\mu) ; \mu \in P_{f}\right\}$ is achieved at a probability $v$ such that $v=\delta_{x}$ with $f(x) \geq 0$ or $v$ is s-affine on a segment $[a, b]$, $\int f d v=0$ and $\int_{a}^{x} f d v>0$ on $(a, b)$ or $\int_{x}^{b} f d v>0$ on $(a, b)$.

Proof of Theorem 2. By Theorem 2.2 of [2], we know that the set of $s$-concave probabilities supported in $K$ is $w^{*}$-compact. Since $f$ is upper semi-continuous, the condition $\int f d \mu \geq 0$ is $w^{*}$-closed, therefore the set $P_{f}$ is $w^{*}$-compact. By application of the Krein-Milman theorem, $\sup \left\{\Phi(\mu) ; \mu \in P_{f}\right\}$ is achieved at a probability $v \in \mathcal{E} \mathrm{xt}\left(\overline{\operatorname{conv}}{ }^{*} P_{f}\right) \subset \mathcal{E} \mathrm{xt}\left(\operatorname{conv} P_{f}\right)$. The result follows by the description of the extreme points of conv $P_{f}$ given in Theorem 1.

As a direct application, we deduce the following generalization of the geometric KLS localization theorem in its more popular form:

Corollary 1. Let $f_{1}, f_{2}$ be two upper semi-continuous non-negative functions on $\mathbb{R}^{n}$ and let $f_{3}, f_{4}$ be two lower semi-continuous non-negative functions on $\mathbb{R}^{n}$. Let $-\infty \leq$ $s \leq \frac{1}{2}$ and $\alpha, \beta>0$. Suppose that $f_{1}^{\alpha} f_{2}^{\beta} \leq f_{3}^{\alpha} f_{4}^{\beta}$ and that for every $a, b \in \mathbb{R}^{n}$, for every s-affine probability v supported by $[a, b]$,

$$
\left(\int f_{1} d v\right)^{\alpha}\left(\int f_{2} d v\right)^{\beta} \leq\left(\int f_{3} d v\right)^{\alpha}\left(\int f_{4} d v\right)^{\beta}
$$

Then for every s-concave probability $\mu$ on $\mathbb{R}^{n}$,

$$
\left(\int f_{1} d \mu\right)^{\alpha}\left(\int f_{2} d \mu\right)^{\beta} \leq\left(\int f_{3} d \mu\right)^{\alpha}\left(\int f_{4} d \mu\right)^{\beta} .
$$

Proof. By adding a small constant to $f_{3}$, we may assume that $f_{3}>0$ on $\mathbb{R}^{n}$. Let $\mu$ be an $s$-concave compactly supported probability in $\mathbb{R}^{n}$ and denote its support by $K$. Define

$$
f=f_{1}-\left(\frac{\int f_{1} d \mu}{\int f_{3} d \mu}\right) f_{3}, \quad \Phi(\theta)=\left(\frac{\int f_{1} d \mu}{\int f_{3} d \mu}\right)^{\alpha / \beta}\left(\int f_{2} d \theta\right)-\left(\int f_{4} d \theta\right)
$$


for every probability $\theta \in \mathcal{P}(K)$ and $P_{f}$ as in Theorem 1. By the assumptions on $f_{1}$, $f_{2}, f_{3}$ and $f_{4}$, the functions $f$ and $\Phi$ are upper semi-continuous and $\Phi$ is affine. Since $\mu \in P_{f}$, by Theorem 2, there exists a probability $v$ which is either a Dirac or $s$-affine and supported by a segment $[a, b]$ such that $\Phi(\mu) \leq \Phi(v)$ and $\int f d v \geq 0$. This gives

$$
\begin{aligned}
\left(\frac{\int f_{1} d \mu}{\int f_{3} d \mu}\right)^{\alpha / \beta} \int f_{2} d \mu-\int f_{4} d \mu & \leq\left(\frac{\int f_{1} d \mu}{\int f_{3} d \mu}\right)^{\alpha / \beta} \int f_{2} d \nu-\int f_{4} d v \\
& \leq\left(\frac{\int f_{1} d \nu}{\int f_{3} d v}\right)^{\alpha / \beta} \int f_{2} d v-\int f_{4} d v \leq 0
\end{aligned}
$$

By a standard approximation this gives the result for any $s$-concave probability in $\mathbb{R}^{n}$.

\section{Proof of Theorem 1}

Obviously the Dirac measures at points $x$ such that $f(x) \geq 0$ are extreme points of conv $P_{f}$ thus, from now on, we describe the extreme points of conv $P_{f}$ which are not Dirac measures.

In steps $1-3$ we fix a measure $v$, extreme in $\operatorname{conv} P_{f}$, which is not a Dirac measure and prove that it satisfies the properties quoted in (2) of Theorem 1 . We denote by $G$ the least affine subspace which contains the support of $v$.

Step 1: $\operatorname{dim} G=1$.

Proof. If $\operatorname{dim} G \geq 2$ there exists $x_{0}$ in the relative interior of supp $v$ and a two-dimensional subspace of $\mathbb{R}^{n}, E$ such that $x_{0}+E \subset G$. For all $u \in S_{1}(E)$, the unit circle in $E$, let $H_{u}=\left\{x \in G ;\left\langle x-x_{0}, u\right\rangle=0\right\}, H_{u}^{+}=\left\{x \in G ;\left\langle x-x_{0}, u\right\rangle \geq 0\right\}$ and $H_{u}^{-}=\{x \in$ $\left.G ;\left\langle x-x_{0}, u\right\rangle \leq 0\right\}$ and define $\phi: S_{1}(E) \rightarrow \mathbb{R}$ by $\phi(u)=\int_{H_{u}^{+}} f d v-\left(\int f d v\right) / 2$. For all $u \in S_{1}(E), H_{u}$ is a hyperplane in $G$, hence $v\left(H_{u}\right)=0$. This implies that $\phi(-u)=-\phi(u)$ and that $\phi$ is continuous. Hence there exists $u_{0} \in S_{1}(E)$ such that $\phi\left(u_{0}\right)=0$. By the choice of $x_{0}$, it is clear that $v\left(H_{u_{0}}^{+}\right)>0$ and $v\left(H_{u_{0}}^{-}\right)>0$, thus we may define $v_{1}=v_{\mid H_{u_{0}}^{+}} / v\left(H_{u_{0}}^{+}\right)$and $v_{2}=v_{\mid H_{u_{0}}^{-}} / v\left(H_{u_{0}}^{-}\right)$. Then $v=v\left(H_{u_{0}}^{+}\right) v_{1}+v\left(H_{u_{0}}^{-}\right) v_{2}$ with $v_{1}$ and $v_{2} \in P_{f} \backslash\{\nu\}$, which means that $\nu$ is not extreme in conv $P_{f}$.

From step 1 and Borell's characterization, the support of $v$ is a segment $[a, b], d v=$ $\psi d m$, where $m$ is the Lebesgue measure on $[a, b], 0 \leq \psi \in L_{\mathrm{loc}}^{1}\left(\mathbb{R}^{n}, d m\right)$ and $\psi$ is $\gamma$-concave with $\gamma=s /(1-s)$. We have $-\infty \leq s \leq \frac{1}{2}$, hence $\gamma \in[-1,1]$.

Step 2: $\int f d v=0$ and $\int_{a}^{x} f d v>0$ on $(a, b)$ or $\int_{x}^{b} f d v>0$ on $(a, b)$.

Proof. Notice that the function $x \mapsto \int_{a}^{x} f d \nu$ is continuous on $[a, b]$. If there exists $c \in$ $(a, b)$ such that $\int_{a}^{c} f d v=0$, define $v_{1}=v_{[[a, c]} / \nu([a, c])$ and $v_{2}=v_{[[c, b]} / v([c, b])$, then $v=v([a, c]) v_{1}+v([c, b]) v_{2}$ with $v_{1}$ and $v_{2} \in P_{f} \backslash\{v\}$. This means that $v$ is not extreme in conv $P_{f}$. If $\int f d v>0$, then there exists $c \in(a, b)$ with $\int_{a}^{c} f d v=\left(\int f d v\right) / 2>0$. Defining $v_{1}$ and $v_{2}$ in the same way we get the same result.

Step 3: $v$ is s-affine. 
Proof. By step 2 we may assume that $\int_{a}^{x} f d v>0$, for all $x \in(a, b)$. Let $c \in(a, b)$, $u=(b-a) /\|b-a\|_{2}$ and for $\alpha \in \mathbb{R}$, define $\varphi_{\alpha}:[a, b] \rightarrow \mathbb{R}$ by $\varphi_{\alpha}(x)=(\psi(c) / 2)(1+$ $\gamma \alpha\langle x-c, u\rangle)_{+}^{1 / \gamma}$ extending it by continuity for $\gamma=0$. It is clear that $\varphi_{\alpha}$ is $\gamma$-affine on $[a, b]$. We consider the following measures with support included in $[a, b]$ :

$$
d \mu_{\alpha}=\left(\psi-\varphi_{\alpha}\right)_{+} d m, \quad d v_{\alpha}=\inf \left(\psi, \varphi_{\alpha}\right) d m .
$$

Since $\gamma \in[-1,1], \psi$ is $\gamma$-concave and $\varphi_{\alpha}$ is $\gamma$-affine on $[a, b]$, we get from Lemma 1 that $\left(\psi-\varphi_{\alpha}\right)_{+}$is $\gamma$-concave so $\mu_{\alpha}$ is $s$-concave. It is clear that $\inf \left(\psi, \varphi_{\alpha}\right)$ is also $\gamma$ concave so $v_{\alpha}$ is also $s$-concave. By Lebesgue's theorem, the function $\alpha \mapsto \int f d \nu_{\alpha}$ is continuous on $\mathbb{R}$ and

$$
\lim _{\alpha \rightarrow-\infty} \int f d v_{\alpha}=\int_{a}^{c} f d v>0, \quad \lim _{\alpha \rightarrow+\infty} \int f d v_{\alpha}=\int_{c}^{b} f d v<0
$$

since $\int f d v=0$. Hence there exists $\alpha_{0}$ such that $\int f d v_{\alpha_{0}}=0$. Since $v=\mu_{\alpha_{0}}+v_{\alpha_{0}}$ this gives $\int f d \mu_{\alpha_{0}}=0$ and taking $\lambda=v_{\alpha_{0}}([a, b]) \in(0,1)$, we get $v=(1-\lambda) v_{1}+\lambda \nu_{2}$ where $v_{1}=\mu_{\alpha_{0}} /(1-\lambda)$ and $v_{2}=v_{\alpha_{0}} / \lambda$. The probabilities $v_{1}$ and $v_{2}$ belong to $P_{f}$ and since $v$ is extreme, we deduce that $\nu_{1}=\nu_{2}=v$ which means that $\psi=\varphi_{\alpha_{0}} / \lambda$ and $v$ is $s$-affine.

In step 4 we prove that a probability satisfying properties (2) of Theorem 1 is extreme in $\operatorname{conv} P_{f}$.

Step 4: if a probability $v$ is s-affine on a segment $[a, b] \subset \mathbb{R}^{n}, \int f d v=0$ and $\int_{a}^{x} f d v>$ $\overline{0 \text { on }}(a, b)$ or $\int_{x}^{b} f d v>0$ on $(a, b)$, then $v$ is extreme in $\operatorname{conv} P_{f}$.

Proof. We define $F:[a, b] \rightarrow \mathbb{R}$ by $F(x)=\int_{a}^{x} f d \nu$, we may and do assume that $F>0$ on $(a, b)$. We denote by $\psi$ the density of $v$ with respect to the Lebesgue measure $m$ on $[a, b]$. It is a $\gamma$-affine function on $[a, b]$. Suppose that $v$ is the convex combination of measures $\mu_{1}, \ldots, \mu_{p} \in P_{f} \backslash\{v\}$ :

$$
v=\sum_{i=1}^{p} \lambda_{i} \mu_{i}, \quad 0<\lambda_{i} \leq 1, \quad \sum_{i=1}^{p} \lambda_{i}=1 .
$$

For all $i=1, \ldots, p, \int f d \mu_{i} \geq 0$, since $\mu_{i} \in P_{f}$, and by hypothesis on $v, \int f d v=$ $0=\sum_{i=1}^{p} \lambda_{i} \int f d \mu_{i}$ so $\int f d \mu_{i}=0$. By (1), for each $i=1, \ldots, p, \mu_{i}$ is absolutely continuous with respect to $v$ and since $\mu_{i} \in P_{f}$, it has a $\gamma$-concave density $\psi_{i}$ with respect to $m$ on $[a, b]$. Therefore, if we denote by $\rho_{i}$ the density of $\mu_{i}$ with respect to $\nu$, we have

$$
\sum_{i=1}^{p} \lambda_{i} \rho_{i}=1 \quad \text { on }[a, b] \quad \text { and } \quad \rho_{i}=\frac{d \mu_{i}}{d \nu}=\frac{\psi_{i}}{\psi}
$$

is the quotient of a $\gamma$-concave function by a $\gamma$-affine function. It is easily checked that these properties imply that $\rho_{i}$ is quasi-concave, non-negative and continuous on its support. Moreover a quasi-concave function on a segment is either monotone or first non-decreasing then non-increasing on this segment. Since $\sum_{i=1}^{p} \lambda_{i} \rho_{i}=1$ on $[a, b]$, at least one of the functions $\rho_{i}$ which are non-zero at $a$ is non-increasing in a neighbourhood 
of $a$. We may assume that one of these functions is $\rho_{1}$. Hence $\rho_{1}$ is non-increasing on its support $[a, c]$, where $c \in(a, b]$. Integrating by parts, we get

$$
\int f d \mu_{1}=0=\int_{a}^{c} \rho_{1} d F=\left[\rho_{1} F\right]_{a}^{c}-\int_{a}^{c} F d \rho_{1}=\rho_{1}(c) F(c)-\int_{a}^{c} F d \rho_{1} .
$$

Since $\rho_{1}(c) F(c) \geq 0, \rho_{1}$ is non-increasing and $F>0$ on $(a, c)$, this implies that $\rho_{1}$ is constant on $[a, c]$ and $F(c)=0$. Therefore $c=b$ and since $d \mu_{1}=\rho_{1} d \nu$ with $\mu_{1}$ and $v$ probabilities on $[a, b], \rho_{1}=1$ on $[a, b]$ hence $\mu_{1}=v$; this is absurd.

This ends the proof of Theorem 1.

\section{Generalization to Several Constraints}

We do not know the exact characterization of the extreme points of the set of $s$-concave probabilities satisfying several constraints but we can still establish the following generalization of Theorem 1.

Theorem 3. Let $n$ be a positive integer, let $K$ be a compact convex set in $\mathbb{R}^{n}$, let $p \in\{1, \ldots, n\}$ and let $-\infty \leq s \leq 1 /(p+1)$. For $f=\left(f_{1}, \ldots, f_{p}\right): K \rightarrow \mathbb{R}^{p}$, with $f_{i}$ upper semi-continuous functions, we denote by $P_{f}$ the set of $s$-concave probabilities supported in $K$ satisfying $\int f_{i} d \mu \geq 0, \forall i$. Let $v$ be an extreme point of $\operatorname{conv} P_{f}$ and denote by $G$ the least affine subspace which contains its support. Then $\operatorname{dim} G \leq p$ and if $\operatorname{dim} G=p$, then $v$ is $s$-affine on its support, $\int f d v=0$ and for all $x_{0}$ in the relative interior of $\operatorname{supp}(v)$, for all $u$ in the unit sphere of $G, \int_{\left\langle x-x_{0}, u\right\rangle \geq 0} f d v \neq 0$. Moreover, if $\Phi: \mathcal{P}(K) \rightarrow \mathbb{R}$ is a convex upper semi-continuous function then $\sup _{\mu \in P_{f}} \Phi(\mu)$ is achieved at an extreme point of $\operatorname{conv} P_{f}$ as described above.

Proof. Fix a measure $v$, extreme in $\operatorname{conv} P_{f}$ and let $G$ be the least affine subspace which contains the support of $v$, we can assume that $\operatorname{dim} G \geq p$.

Step 1: $\operatorname{dim} G=p$.

Proof. If $\operatorname{dim} G \geq p+1$ there exists $x_{0}$ in the relative interior of $\operatorname{supp} v$ and a $(p+1)$ dimensional subspace of $\mathbb{R}^{n}, E$ such that $x_{0}+E \subset G$. For all $u \in S_{p}(E)$, the unit sphere in $E$, let $H_{u}=\left\{x \in G ;\left\langle x-x_{0}, u\right\rangle=0\right\}, H_{u}^{+}=\left\{x \in G ;\left\langle x-x_{0}, u\right\rangle \geq 0\right\}$ and $H_{u}^{-}=\{x \in$ $\left.G ;\left\langle x-x_{0}, u\right\rangle \leq 0\right\}$ and define $\phi: S_{p}(E) \rightarrow \mathbb{R}^{p}$ by $\phi(u)=\int_{H_{u}^{+}} f d v-\left(\int f d v\right) / 2$. For all $u \in S_{p}(E), H_{u}$ is a hyperplane in $G$, hence $v\left(H_{u}\right)=0$. This implies that $\phi(-u)=-\phi(u)$ and that $\phi$ is continuous. Hence, from Borsuk's theorem, there exists $u_{0} \in S_{p}(E)$ such that $\phi\left(u_{0}\right)=0$. By the choice of $x_{0}$, it is clear that $v\left(H_{u_{0}}^{+}\right)>0$ and $v\left(H_{u_{0}}^{-}\right)>0$, thus we may define $v_{1}=v_{\mid H_{u_{0}}^{+}} / v\left(H_{u_{0}}^{+}\right)$and $v_{2}=v_{\mid H_{u_{0}}^{-}} / v\left(H_{u_{0}}^{-}\right)$. Then $v=v\left(H_{u_{0}}^{+}\right) v_{1}+v\left(H_{u_{0}}^{-}\right) v_{2}$ with $v_{1}$ and $v_{2} \in P_{f} \backslash\{v\}$, which means that $v$ is not extreme in conv $P_{f}$.

Step 2: for all $x_{0}$ in the interior of $\operatorname{supp}(v)$, for all $u$ in the unit sphere of $G, \int_{\left\langle x-x_{0}, u\right\rangle \geq 0} f d v$ $\neq 0$ and $\int f d v=0$. 
Proof. Assume first that there exists $x_{0}$ in the interior of $\operatorname{supp}(v)$, and $u$ in the unit sphere of $G$, such that $\int_{\left\langle x-x_{0}, u\right\rangle \geq 0} f d v=0$. We define $H^{+}=\left\{x \in G ;\left\langle x-x_{0}, u\right\rangle \geq 0\right\}$, $H^{-}=\left\{x \in G ;\left\langle x-x_{0}, u\right\rangle \leq 0\right\}, v_{1}=v_{\mid H^{+}} / v\left(H^{+}\right)$and $v_{2}=v_{\mid H^{-}} / v\left(H^{-}\right)$. Then $v=v\left(H^{+}\right) v_{1}+v\left(H^{-}\right) v_{2}$ with $v_{1}$ and $v_{2} \in P_{f} \backslash\{v\}$, which means that $v$ is not extreme in $\operatorname{conv} P_{f}$.

Now we prove that $\int f d v=0$. Assume that $\int f d v \neq 0$. We identify $G$ with $\mathbb{R}^{p}$ and we denote by $e_{p+1}$ a vector orthogonal to $G$ in $\mathbb{R}^{n}$. For every $u \in S^{p}$, the unit sphere of $G \oplus \mathbb{R} e_{p+1}$, let $H_{u}^{+}=\left\{x \in G ;\left\langle x-e_{p+1}, u\right\rangle \geq 0\right\}$ and $H_{u}^{-}=\left\{x \in G ;\left\langle x-e_{p+1}, u\right\rangle \leq\right.$ $0\}$. This is a parametrization of the half-spaces of $G$ by $S^{p}$. As in step 1, we define $\phi: S^{p} \rightarrow \mathbb{R}^{p}$ by $\phi(u)=\int_{H_{u}^{+}} f d v-\left(\int f d v\right) / 2$. The same use of Borsuk's theorem gives a vector $u_{0} \in S^{p}$ such that $\phi\left(u_{0}\right)=0$. We conclude as in step 1 that $v$ is not extreme in conv $P_{f}$.

Observe that the first part is also valid when $\operatorname{dim} G<p$ while in the second part, the use of Borsuk's theorem requires $\operatorname{dim} G=p$.

Step 3: $v$ is s-affine.

Proof. By the characterization of Borell and steps 1 and 2, $v$ is absolutely continuous with respect to the Lebesgue measure $m$ on $G, d \nu / d m=\psi$ is $\gamma$-concave on $G$ with $\gamma=s /(1-s p)$ and $\int f d v=0$. As in step 2 , we identify $G$ with $\mathbb{R}^{p}$, we denote by $e_{p+1}$ a vector orthogonal to $G$ in $\mathbb{R}^{n}$ and by $S^{p}$ the unit sphere of $G \oplus \mathbb{R} e_{p+1}$. Let $c$ be within the relative interior of $\operatorname{supp}(v)$. For every $u \in S^{p}$ such that $\left\langle u, e_{p+1}\right\rangle \neq 0$, we define a $\gamma$-affine function $\varphi_{u}: K \rightarrow \mathbb{R}$ by

$$
\varphi_{u}(x)=\frac{\psi(c)}{2}\left(1+\gamma \frac{\langle x-c, u\rangle}{\left\langle e_{p+1}, u\right\rangle}\right)_{+}^{1 / \gamma},
$$

extending it by continuity for $\gamma=0$. We also define $\phi: S^{p} \rightarrow \mathbb{R}^{p}$ by

$$
\phi(u)=\left\{\begin{array}{lll}
\int f\left(\psi-\varphi_{u}\right)_{+} d m & \text { if } & \left\langle u, e_{p+1}\right\rangle>0, \\
\int f \inf \left(\psi, \varphi_{u}\right) d m & \text { if } & \left\langle u, e_{p+1}\right\rangle<0, \\
\int_{\langle x-c, u\rangle \geq 0} f d v & \text { if } & \left\langle u, e_{p+1}\right\rangle=0 .
\end{array}\right.
$$

It is easily checked that $\phi$ is continuous on $S^{p}$ and $\phi(u)+\phi(-u)=\int f d v=0$. Hence, from Borsuk's theorem, there exists $u_{0} \in S_{p}$ such that $\phi\left(u_{0}\right)=0$. From step 2, $\left\langle u_{0}, e_{p+1}\right\rangle \neq 0$. Taking $\lambda=\int \inf \left(\psi, \varphi_{u_{0}}\right) d m \in(0,1)$, we define $v_{1}$ and $v_{2}$ by $d v_{1} / d m=$ $\left(\psi-\varphi_{u_{0}}\right)_{+} /(1-\lambda)$ and $d \nu_{2} / d m=\inf \left(\psi, \varphi_{u_{0}}\right) / \lambda$. We have $-\infty \leq s \leq 1 /(p+1)$, hence $\gamma=s /(1-s p) \in[-1,1]$. By Lemma 1 , it is clear that $\nu_{1}, \nu_{2} \in P_{f}$. Since $v=(1-\lambda) v_{1}+\lambda v_{2}$ and $v$ is extreme, we deduce that $v_{1}=v_{2}=v$ which means that $\psi=\varphi_{u_{0}} / \lambda$ and $v$ is $s$-affine.

The proof of the part with the function $\Phi$ is exactly the same as in Theorem 2. This ends the proof of Theorem 3 .

\section{Acknowledgements}

We thank Professors Gilles Godefroy and Bernard Maurey for many useful discussions. 


\section{References}

1. S. G. Bobkov, Remarks on the growth of $L p$-norms of polynomials, in Geometric Aspects of Functional Analysis, pp. 27-35, Lecture Notes in Mathematics, 1745, Springer-Verlag, Berlin, 2000.

2. C. Borell, Convex measures on locally convex spaces, Ark. Mat. 12 (1974), 239-252.

3. C. Borell, Convex set functions in $d$-space, Period. Math. Hungar. 6 (1975), 111-136.

4. A. Carbery and J. Wright, Distributional and $L q$ norm inequalities for polynomials over convex bodies in $\mathbb{R}^{n}$. Math. Res. Lett. 8(3) (2001), 233-248.

5. O. Guédon, Kahane-Khinchine type inequalities for negative exponent, Mathematika 46(1) (1999), 165173.

6. R. Kannan, L. Lovász and M. Simonovits, Isoperimetric problems for convex bodies and a localization lemma, Discrete Comput. Geom. 13(3-4) (1995), 541-559.

7. L. Lovász and M. Simonovits, Random walks in a convex body and an improved volume algorithm, Random Structures Algorithms 4(4) (1993), 359-412.

8. F. Nazarov, M. Sodin and A. Volberg, The geometric Kannan-Lovász-Simonovits lemma, dimension-free estimates for the distribution of the values of polynomials, and the distribution of the zeros of random analytic functions (in Russian). Algebra Anal. 14(2) (2002), 214-234.

Received March 22, 2002, and in revised form April 4, 2003. Online publication December 19, 2003. 